\title{
ELABORACIÓN DE UNA LISTA DE COMPROBACIÓN CON BASE A LAS NORMATIVAS ESTABLECIDOS POR LOS ORGANISMOS DE CONTROL FRENTE A LOS AGENTES BIOLÓGICOS
}

\section{Elaboration of a Checklist Based on the Regulations Established by the Control Bodies Against Biological Agents}

Pavel Omar Defranc Balanzategui, MsC. Instituto Superior Universitario de Formación Profesional Administrativa y Comercial, Ecuador https://orcid.org/0000-0002-2796-9829 pavel.defranc@formacion.edu.ec
Abdón Isaac Arellano Valdiviezo, Dr. Instituto Superior Universitario de Formación Profesional Administrativa y Comercial, Ecuador https://orcid.org/0000-0003-0304-8301 abdon.arellano@formacion.edu.ec
Roberto Bolívar Oviedo Quiñonez, Ing. Consultor en Prevención de Riesgos Laborales, Ecuador

https://orcid.org/0000-0003-1311-2573 rovertoviedo_q@hotmail.com

Palabras claves: Lista de Comprobación, Pandemia, Agentes Biológicos, Protocolos de Bioseguridad.

Recibido: 22 de febrero de 2021

Keywords: Checklist, Pandemic, Biological Agents, Biosafety Protocols.

Aceptado: 29 de abril de 2021

\section{RESUMEN}

Ante los impactos negativos de la pandemia de COVID-19 en el mundo, Latinoamérica y, particularmente, en Ecuador se han decretado múltiples normas y restricciones para impedir su impetuosa propagación; sin embargo, no existía una lista de comprobación de los protocolos para medir su cumplimiento, por lo que se propone una lista de comprobación con base a las normativas establecidos por los organismos de control frente a los agentes biológicos, dirigida a verificar el grado de cumplimiento de los criterios técnicos-legales establecidos en los protocolos de bioseguridad, acorde a las exigencias de los entes de control. La cual se ha aplicado en instituciones y empresas del sector público y privado al momento de realizar la evaluación.

\begin{abstract}
Given the negative impacts of the COVID-19 pandemic in the world, Latin America and, particularly, in Ecuador, multiple regulations and restrictions have been decreed to prevent its impetuous spread; However, there was no checklist of protocols to measure compliance, so a checklist is proposed based on the regulations established by control bodies against biological agents, aimed at verifying the degree of compliance of the technical-legal criteria established in the biosafety protocols, according to the requirements of the control entities. This has been applied in institutions and companies of the public and private sectors at the time of the evaluation.
\end{abstract}




\section{INTRODUCCIÓN}

El 31 de diciembre del 2019, el municipio de Wuhan en la provincia de Hubei, China, informó un grupo de casos de neumonía con etiología desconocida. Para el 9 d enero del 2020, el centro Chino para el Control y la Prevención de enfermedades identificó un nuevo coronavirus (2019-nCoV) como el agente causante de este brote.

El 30 de enero con más de 9700 casos confirmados en China y 106 casos confirmados en otros 19 países, el Director general de la Organización Mundial de la Salud, declaró el brote como una emergencia de salud pública de importancia internacional (ESPII), aceptando la recomendación del Comité de Emergencia del Reglamento Sanitario Internacional (2005).

Entre el 31 de diciembre del 2019 y el 3 de septiembre del 2020, se notificaron un total de 25.208 .690 casos confirmados por el laboratorio de infección por el 2019-nCOV 188 países, con 867.219 fallecimientos según Organización Panamericana de la Salud www.paho.org

Ecuador, expectante de cómo se movilizaba el virus en el mundo le fue imposible detener la llegada del mismo a nuestro suelo, y, el lunes 27 de enero del 2020 surge la primera sospecha de ingreso del virus a nuestro medio por parte de un ciudadano proveniente de España; posteriormente, el 29 de febrero se confirma el primer paciente detectado mediante pruebas con COVID-19, tras el aumento de casos dentro del país, además de la región latinoamericana y el mundo.

El 14 de marzo de 2020, se conforma la primera reunión del COE nacional, conformada por los distintos representantes del Gobierno Nacional, donde empiezan un sinnúmeros de prohibiciones, como: el no ingreso de personas del Exterior; cumplimiento de aislamientos preventivos; obligación de limpiezas a transportes cada tres horas, habilitación de laboratorios privados para realizar exámenes de prueba de COVID; restricción en los gimnasios, cines, teatros, festividades, conciertos, funciones de circos, y de personas en espacios públicos; la implementación de protocolos de bioseguridad frente a riesgos biológicos; entre otras.

La noche del lunes 16 de marzo, el Presidente de la República, Lenin Moreno Garcés, a través de una cadena nacional, declaró el Estado de Excepción en todo el territorio ecuatoriano, con el propósito de contener la transmisión del coronavirus (COVID-19); posteriormente, se oficializa una cuarentena obligatoria que se extendería hasta el 12 de septiembre del año en curso. Fuente «Gobierno decreta emergencia sanitaria en Ecuador por covid-19». El Comercio. Consultado el 23 de marzo de 2020.

Dentro todo este proceso de crecimiento del COVID 19 en el país continuaron surgiendo un sinnúmero de requerimientos legales que debían aplicarse a todas las organizaciones, para poder operar frente a esta emergencia, una de estas fue la declarada en la mesa técnica del COE Cantonal Guayaquil donde decide que las empresas deberán realizar una auditoría externa de seguridad de salud ocupacional, en el término de 30 días, posteriores a la emisión del permiso.

Lo anterior, conllevó a cuestionarnos que: si bien el Gobierno mediante su COE Nacional y los diferentes COE Cantonales habían dictado un sinnúmero de norma a las empresas, no existía una lista de comprobación de los protocolos en el cual podamos medir el cumplimiento de los mismos, es así que proponemos la lista de comprobación con base a las normativas establecidos por los organismos de control frente a los agentes biológicos. Cuyo objetivo estaba dirigido a: Elaborar una lista de comprobación que permita verificar el grado de cumplimiento de los criterios técnicos-legales establecidos en los protocolos de bioseguridad, acorde a las exigencias emitidas por los entes de control.

El alcance de la Lista de comprobación de protocolos de bioseguridad, ha sido desarrollado para el uso de instituciones y empresas del sector público y privado, aplicable a nivel nacional, donde se deberá contemplar los requerimientos legales de cada GAD al momento de realizar la evaluación.

\section{DESARROLLO}

El diseño de la lista de comprobación se basa en la metodología elaborada por los autores, donde se consideraron las siguientes etapas para su construcción con los siguientes pasos:

1. Determinar el propósito y alcance de la Lista: Evaluar los protocolos de bioseguridad empresarial exigidos por los entes de control. 
2. Decidir sobre el tipo de instrumento: Documento tipo cuestionario de administración indirecta, debido a que la aplicación la realizará una persona especializada en Seguridad y salud laboral, quien es la que formula las preguntas del cuestionario o realiza la observación con base al mismo.

3. Analizar la información contenida dentro de la teoría, para luego elaborar las dimensiones, subdimensiones y los ítems que conformarán la lista de comprobación.

4. Para la lista de comprobación se ha utilizado una escala simple con 3 niveles de respuesta (escala nominal): cumple, indica que satisface el criterio enunciado; no cumple, indicaausencia de cierta característica, y no aplica significa que el criterio no se emplea.

5. Conceptualizar las preguntas: Para la conceptualización de las dimensiones y los subdimensiones de la lista de comprobación se tomó como base los criterios emitidos y pedidos por los entes de control, así como la normativa legal vigente en el Ecuador con respecto a Seguridad y Salud en el Trabajo.

6. Operacionalización de las preguntas: Esta etapa se caracterizó por la elaboración de las preguntas, basados en las variables identificadas, desglosándolos en dimensión y subdimensiones, representadas en una matriz. El tipo de preguntas que se utilizó fueron preguntas cerradas, que se caracterizan por establecer previamente alternativas de respuesta, para medirlas se utilizó una medición de tipo ordinal, donde se establecieron tres categorías de forma cuantitativa, permitiendo ordenar las respuestas en función de mayor a menor atributo a medir.

7. Asignar porcentajes de cumplimiento o ejecución, dependiendo del número de dimensiones e ítems a evaluar; para la asignación de porcentajes de calificación se utilizó la estrategia de grupo criterio (empírica) (Aiken, 2003) y una ponderación a cada una de las estructuras de forma lineal, establecidas sobre un total de 79 ítems $y$, se resume de la siguiente manera:

Las tres dimensiones a evaluar, desarrolladas en la lista de comprobación son:
* Estructura Organizativa $17.72 \%$
* Estructura Técnica $69.62 \%$
* Estructura Vigilancia Médica $12.66 \%$

a) La Estructura Organizativa consta de dos parámetros, con un total de 14 preguntas.

1) Medidas Organizativas: Consta de 8 preguntas.

2) Proveedores y Clientes: Consta de 6 preguntas.

b) La Estructura Técnica consta de nueve parámetros, con un total de 55 preguntas.

1) Evaluación del Riesgo Biológico: Consta de 2 preguntas.

2) Señalización: Consta de 8 preguntas.

3) Transporte de trabajadores y carga: Consta de 4 preguntas.

4) Almacenamiento, manipulación de insumos y productos: Consta de 5preguntas.

5) Distancia física y Aforo: Consta de 6 preguntas.

6) Medidas Locativas: Consta de 10 preguntas.

7) Residuos: Consta de 7 preguntas.

8) Limpieza y desinfección: Consta de 10 preguntas.

9) Equipos de protección personal y colectiva: Consta de 3 preguntas.

c) La Estructura Vigilancia Médica que consta de dos parámetros, con un total de 10 preguntas.

1) Evaluación Médica: Consta de 3 preguntas.

2) Control y Seguimiento: Consta de 7 preguntas

La lista de comprobación refleja los contenidos de los protocolos de bioseguridad exigidos por los entes de control, considerando la dimensión, subdimensiones y sus variables, y estas se presentan a manera de preguntas. Además, se elaboró las instrucciones para aplicar la lista de comprobación de recolección de datos y sumar los respectivos puntajes para su interpretación.

Cabe resaltar que este prototipo es solo una aproximación de operacionalización de las variables a intervenir (ítems), el mismo que debe ser sometido para su validez a una prueba piloto, ya que su construcción por un grupo de expertos, brinda la valía de su contenido y ampliación. 


\section{MANEJO DE RESULTADOS}

Una vez diligenciada la evaluación, se realiza la sumatoria, para poder tabular todos los criterios evaluados por cada dimensión y subdimensión, con el valor correspondiente a las alternativas presentadas, las cuales se establecen a continuación:

A. Si cumple con los criterios establecidos le asignará el puntaje y porcentaje correspondiente.

B. No cumple, con los criterios establecidos le asignará el puntaje y porcentaje correspondiente.

C. No aplica los criterios establecidos, no se considerará para la asignación de puntaje, por tanto, no entrará en el cálculo.

Terminado este procedimiento, se realiza la sumatoria de los ítems de los subdimensiones de cada dimensión.

Para establecer el promedio parcial de la estructura organizativa, de la estructura técnica y de la estructura vigilancia médica, se calculará de la siguiente manera:

A. Para obtener el promedio parcial del componente "estructura organizativa", se sumará el total de los ítems cumplidos de los subdimensiones y se dividirá este resultado para el total de ítems evaluados aplicables de los subdimensiones multiplicado por 100.

B. Para obtener el promedio parcial del componente "estructura técnica", se sumará el total de los ítems cumplidos de las subdimensiones y se dividirá este resultado para el total de ítems evaluados aplicables de los subdimensiones multiplicado por 100.

C. Para obtener el promedio parcial del componente estructura vigilancia médica, se sumará el total de los ítems cumplidos de las subdimensiones y se dividirá este resultado para el total de ítems evaluados aplicables de las subdimensiones multiplicado por 100.

Cabe resaltar que la lista de comprobación permite identificar los resultados de la evaluación de las dimensiones y subdimensiones, permitiendo identificar e interpretar los resultados de forma parcial de acuerdo a las necesidades de la empresa y poder intervenir en cada una de ellas.

Para establecer el promedio ponderado de las dimensiones, se debe multiplicar el resultado parcial de cada dimensión por su ponderación (A. Estructura Organizativa (E.O.) 0,1772; B. Estructura Técnica (E.T.) 0,6962 y C. Estructura Vigilancia Médica (E.V.M.) 0,1262).

La suma de cada resultado ponderado de las estructuras dará el resultado total que se denomina el índice de cumplimiento global (ICG), para lo cual se establece la siguiente expresión matemática:

$$
\begin{aligned}
\text { ICG } & =\left\{\left[\left(\frac{N^{\circ} \text { Total ítems cumplidos de la E.0. }}{N^{\circ} \text { Total de ítems aplicables de la E.0. }} \times 100\right) \times 0,1772\right]\right. \\
& +\left[\left(\frac{N^{\circ} \text { Total ítems cumplidos de la E.T. }}{N^{\circ} \text { Total de ítems aplicables de la E.T. }} \times 100\right) \times 0,6962\right] \\
& \left.+\left[\left(\frac{N^{\circ} \text { Total ítems cumplidos de la E.V.M. }}{N^{\circ} \text { Total de ítems aplicables de la E.V.M. }} \times 100\right) \times 0.1262\right]\right\}
\end{aligned}
$$

EI ICG es la cuantificación final de la lista de comprobación que evalúa el protocolo, lo cual permitirá analizar y establecer qué aspectos de las dimensiones requieren mejorarse, ya sea en sus componentes y subcomponentes o en su totalidad. Una vez realizado el cálculo de los promedios e interpretados los resultados, se deberá establecer un plan de acción, en el que se analice la situación, planteen alternativas factibles en tiempos establecidos y se definan responsables, con la finalidad de mejorar, mantener y reforzar los protocolos de bioseguridad.

\section{INTERPRETACIÓN DEL RESULTADO DE LA EVALUACIÓN}

Los rangos de calificación han sido establecidos bajo el libre criterio de los autores (Aiken, 2003), para lo cual se estableció una escala de proporción (Padilla, 2007) que comprenden: 


\section{A. DE 90 A 100 PUNTOS: EXCELENTE}

Este resultado indica que las condiciones de trabajo respecto al protocolo de bioseguridad en la empresa son óptimas, lo que evidencia compromiso de la misma, por lo que se debe continuar así y no se debe permitir que decaiga.

\section{B. DE 70 A 89 PUNTOS: BUENO}

Este resultado indica que las condiciones de trabajo respecto al protocolo de bioseguridad en la empresa responden a los requerimientos necesarios, pero se deben revisar los componentes o subcomponentes débiles para reforzarlas y así lograr condiciones óptimas.

\section{DE 30 A 69 PUNTOS: REGULAR}

Este resultado indica que las condiciones de trabajo respecto al protocolo de bioseguridad en la empresa no cumplen con los criterios establecidos, por lo que se debe identificar los puntos débiles y analizar sus causas, con el fin de buscar estrategias para modificar las deficiencias o ausencias de los componentes y obtener mejoras en un tiempo determinado.

\section{DE 0 A 29 PUNTOS: DEFICIENTE}

Este resultado indica que las condiciones de trabajo respecto al protocolo de bioseguridad en la empresa son insuficientes y precarias, por lo que presentan muchas inconsistencias que no le permiten buenos resultados, por los que se debe hacer una nueva planificación enmarcado en un ciclo de mejora continua, para lograr unos mejores resultados.

\section{LISTA DE COMPROVACION CON BASE A LAS NORMATIVAS ESTABLECIDOS POR LOS ORGANISMOS DE CONTROL FRENTE A LOS AGENTES BIOLOGICOS.}

\section{ESTRUCTURA ORGANIZATIVA}

\section{A. MEDIDAS ORGANIZATIVAS}

1. ¿Cuenta con Unidad de Seguridad e Higiene dirigido por un técnico en la materia? 2.- ¿Cuenta con Responsable de la Gestión de Seguridad, Salud en el Trabajo y Gestión Integral de Riesgos?

2. ¿Cuenta con un médico ocupacional para realizar la gestión de salud en el trabajo?

3. ¿Cuenta con Organismos Paritarios de Seguridad e Higiene del Trabajo?

4. ¿Se cuenta con un Reglamento de Higiene y Seguridad?

5. ¿Cuenta con un Protocolo de Bioseguridad de retorno al trabajo frente al COVID 19?

6. ¿Se asigna los recursos necesarios para la implementación del Protocolo de Bioseguridad COVID-19?

7. ¿Se promueve a todos los trabajadores en teletrabajo, el protocolo de lavado y desinfección de manos?

\section{B. PROVEEDORES CLIENTES}

1. ¿Se ha establecido y socializado con los proveedores y clientes los protocolos de bioseguridad regulados por los entes de control y la organización?

2. ¿La organización ha identificado en sus controles, los métodos, rutas, turnos, plazos y lugares predefinidos de los proveedores y/o clientes a fin de reducir las oportunidades de contacto con sus trabajadores y evitar aglomeraciones?

3. ¿Se fomenta el pago con tarjeta, plataformas digitales y facturación electrónica para reducir el uso de dinero en efectivo y la manipulación de facturas u otra documentación física?

4. ¿En caso de tener contacto con dinero en efectivo, facturas u otros documentos, se han establecido y aplicado las normas de higiene acorde a las actividades relacionadas con esos procesos?

5. ¿Los servicios complementarios (alimentación, limpieza y desinfección, vigilancia) dotan y supervisan la utilización de manera obligatoria de mascarilla y gafas de protección para sus actividades?

6. ¿Se realiza la desinfección de llantas y exteriores de los vehículos de los proveedores /clientes previos al ingreso de las instalaciones?

\section{ESTRUCTURA TÉCNICA}

\section{A. EVALUACIÓN DEL RIESGO BIOLÓGICO}


1. ¿Cuenta con una identificación de peligros y evaluación de riesgos en las áreas de trabajo usando el Método PER-COVID-19-ECUADOR u otras metodologías reconocidas a nivel internacional?

2. ¿Se ha identificado las zonas de alto riesgo en los establecimientos de alto contacto, para implementar nuevas medidas de descontaminación y limpieza?

\section{B. SEÑALIZACION}

1. ¿Se ha implementado señalética relacionada al COVID-19 cumpliendo con los criterios de la norma NTEINEN ISO 3864- ¿1 ¿SÍMBOLOS GRÁFICOS, COLORES DE SEGURIDAD Y SEÑALES DE SEGURIDAD?

2. ¿Se ha colocado señalética en zonas críticas para evitar la aglomeración de personas de forma clara y dirigida a los trabajadores /proveedores /usuarios /clientes sobre el distanciamiento físico permitido de 2 metros mínimo?

3. ¿Se ha colocado señalética en un lugar visible al ingreso del local que indique el aforo permitido dentro del establecimiento acorde a la semaforización indicada por el GAD local?

4. ¿Se ha colocado material gráfico en los baños y otros puntos estratégicos del establecimiento que indique la correcta higiene de manos?

5. ¿Se han implementado letreros o avisos promoviendo los hábitos de no tocarse la boca, nariz, ojos, lavado de manos y uso de alcohol?

6. ¿Se han implementado letreros o avisos promoviendo el uso obligatorio de mascarillas y otros EPP adecuados a la actividad laboral?

7. ¿Los contenedores de desechos biológicos han sido colocados en lugares adecuados y cuentan con señalización?

8. ¿Se ha implementado señalización informativa de los números de emergencia 911 u otro número que la organización disponga para manejo ante emergencias por COVID-19?

\section{TRANSPORTE DE TRABAJADORES Y CARGA}

1. ¿Se cumple la capacidad de pasajeros según la fase decretada por el ente de control y/o GAD Local en referencia al status de semáforo?

2. ¿El medio de transporte cuenta con asientos identificados para ser usados y aquellos que se encuentran restringidos?

3. ¿Se realiza registro y medición de temperatura a todos los pasajeros, como una medida antes de abordar el Transporte de la Empresa?

4. ¿Cuenta con un plan integral de desinfección de vehículos, tanto de transporte, pasajeros, carga?

\section{ALMACENAMENTO, MANIPULACION DE INSUMOS Y PRODUCTOS}

1. ¿Se han establecido y se cumplen las medidas de bioseguridad, limpieza y desinfección de insumos / productos durante los procesos de recepción y entrega?

2. ¿Se mantiene un contacto físico reducido durante el movimiento de productos entre personas?

3. ¿Se hace uso de material resistente en los productos terminados ante su manipulación, como sellos, doble funda, etc. que permita un manejo adecuado con el menor riesgo decontaminación?

4. ¿Se prohíbe el uso de recipientes de bebidas para el almacenamiento de productos químicos o aquellos que constituyan un riesgo para las personas?

5. ¿El lugar de almacenamiento de insumos se encuentra identificado de forma clara y se cuenta con registros de control?

\section{E. DISTANCIAMIENTO FÍSICO Y AFORO}

1. ¿Se han organizado e implementado medidas en las áreas de trabajo y áreas comunes con el objeto de evitar aglomeraciones de los trabajadores implementando jornadas flexibles de trabajo?

2. ¿Se han establecido turnos u horarios flexibles de alimentación para garantizar la distancia mínima entre personas?

3. ¿Se está evitando hacer reuniones presenciales y se favorece el uso de herramientas tecnológicas?

4. ¿Los profesionales Técnicos en Seguridad y Salud en el Trabajo, han establecido mecanismos de seguimiento, monitoreo y control de esta actividad (distanciamiento físico y a foro) en todos los sitios de trabajo? 
5. ¿Se cumple el distanciamiento físico mínimo de 2 metros en los comedores?

6. ¿Las organizaciones socializa sus protocolos y mantiene información actualizada en carteleras o por medios digitales que sean de conocimiento de todo el personal? (Esta acción la deberá realizar de manera obligatoria el representante legal o el Técnico en Seguridad y Salud Ocupacional).

\section{F. MEDIDAS LOCATIVAS}

1. ¿Dispone del número suficiente de puntos para el lavado de manos, cumpliendo con los protocolos de distanciamiento?

2. ¿El almacenamiento de ropa u otros artículos de los trabajadores en el centro de trabajo se realiza de manera segura para evitar la transmisión del virus?

3. ¿Dispone del número suficiente de dispensadores de alcohol, gel para limpieza de manos?

4. ¿Cuenta con ventilación que permita la circulación y recambio de aire en espacios cerrados y en aquellas zonas de escasa ventilación?

5. ¿Cuentan con un plan de mantenimiento de los equipos y sistemas de ventilación?

6. ¿Ha limitado la utilización de elementos de uso común como bebedores de agua, dispensadores de agua por contacto, cafeteras, microondas, máquinas dispensadoras de snacks y bebidas?

7. ¿Promueve el frecuente lavado de manos con jabón antibacterial, así como el uso de gelantiséptico o alcohol al $70 \%$ ?

8. ¿Se ha socializado las buenas prácticas preventivas con su personal tales como evitar compartir bebidas, comidas, utensilios, teléfonos, escritorios, oficinas u otras herramientas de trabajo, equipos y demás elementos de posible uso común?

9. ¿Se ha implementado controles para el uso seguro de ascensores (capacidad, limpieza y desinfección)?

10. ¿Se verifica que los trabajadores mantienen la distancia física de al menos 2 metros durante toda su jornada de trabajo?

\section{G. RESIDUOS}

1. ¿Se han instalado y registrado de manera estratégica los contenedores con funda de basura diferenciada para el desecho de mascarillas, guantes o cualquier otro elemento que pudiera estar contaminado?

2. ¿Se realiza la recolección de residuos generados tales como mascarillas, guantes o cualquier otro elemento contaminado debidamente embolsados y tapados, evitando transitar por áreas productivas sensibles a contaminación?

3. ¿Se ha designado, identificado y socializado un lugar específico para el almacenamiento de los residuos generados tales como mascarillas, guantes o cualquier otro elemento contaminado con acceso restringido sólo para personal autorizado y correctamente señalizado?

4. ¿Los contenedores, tachos y fundas de residuos considerados contaminados son desinfectados previo a su uso y después de su recolección con una solución desinfectante?

5. ¿Cumple con una correcta gestión y disposición de envases de detergentes, jabones y desinfectantes?

6. ¿Se hace la entrega de los residuos contaminados a la entidad recolectora autorizada, de manera que se evite el riesgo de contaminación?

7. ¿Los trabajadores que realizan las tareas de limpieza, desinfección y recolección de residuos han sido capacitados y cumplen con las normas establecidas por la organización?

\section{H. LIMPIEZA Y DESINFECCION}

1. ¿Ha implementado medidas de limpieza y desinfección de las instalaciones y equipos de trabajo? Se deben considerar los espacios comunes como pasillos, escaleras incluido sus pasamanos o mangones, escritorios, mostradores, pisos, puertas, muebles en general, teléfonos, computadoras, agarraderas, manijas de puertas.

2. ¿La Organización ha procedido a cambiar los filtros de los sistemas de aire acondicionado con filtros de alta eficiencia (HEPA)?

3. ¿Se cumple con protocolos de desinfección previos al uso de cualquier elemento o herramienta de trabajo?

4. ¿Se promueve el uso de gel antiséptico o alcohol al $70 \%$ después de cada actividad que implique manipulación de objetos, contacto con superficies, atención a clientes internos y externos?

5. ¿En caso de usar vajillas, vasos, cubiertos no desechables, deberán ser desinfectados después de cada uso, así también los manteles, las mesas y sillas?

6. ¿Se han establecido protocolos y frecuencia para desinfección constantemente del Dispensario Médico? 
7. ¿Ha implementado el uso de Pediluvios para desinfección de calzados en zonas de ingreso al centro de trabajo?

8. ¿Los insumos empleados para realizar actividades de limpieza son sometidos a procesos de desinfección periódica?

9. ¿Cuenta con las fichas de seguridad (SDS) de los productos químicos y se encuentran al alcance y socializadas con los trabajadores?

10. ¿Se han rotulado las diluciones preparadas?

\section{EQUIPOS DE PROTECCION PERSONAL Y COLECTIVA}

1. ¿La organización está dotando a sus empleados con los EPP según una evaluación técnica por puesto de trabajo?

2. ¿Se han establecido barreras físicas en las áreas y lugares de trabajo con el objeto de reducir la exposición entre clientes internos y externos?

3. ¿El personal a cargo de la entrada usa durante su turno los siguientes equipos de protección: ¿gafas de seguridad, mascarillas siguiendo las recomendaciones del fabricante en lo que respecta a su vida útil?

\section{ESTRUCTURA VIGILANCIA MÉDICA}

\section{A. EVALUACIÓN MÉDICA}

1. ¿Se realiza el tamizaje de todo el personal previo al retorno de las actividades laborales?

2. ¿Se evalúa a los trabajadores con sintomatología respiratoria para determinar el manejo del paciente de acuerdo al diagnóstico?

3. ¿Se fomenta hábitos de vida saludable con los trabajadores, como la hidratación frecuente, pausas activas y la disminución del consumo de tabaco como medida de prevención?

\section{B. CONTROL Y SEGUIMIENTO}

1. ¿Se establece un procedimiento de seguimiento diario de salud de los trabajadores previo al ingreso hacia las instalaciones de la empresa?

2. ¿Se notifica a la Unidad de Seguridad y Salud Ocupacional y Talento Humano sobre los casos confirmados por COVID 19 de trabajadores identificados de COVID-19?

3. ¿Participa en la investigación de siniestros laborales asociados a casos confirmados de COVID-19 laborales?

4. ¿Se han establecido lineamientos de control de acuerdo a las normas establecidas para los grupos prioritarios y vulnerables?

5. ¿Se está dando orientación, seguimiento y acompañamiento a los trabajadores, incluidos los de aislamiento preventivo?

6. ¿Dispone de una zona segura para aislamiento de trabajadores en el caso de identificar sospecha de COVID-19?

7. ¿Cuenta con la asesoría del Médico Ocupacional o Psicólogo para atender las necesidades de salud mental de los trabajadores o colaboradores, incluidos los casos de aislamiento social o trabajo en casa?

La guía que se ha pormenorizado y presentado metodológicamente se encuentra en fase se aplicación en diferentes instituciones y empresas del territorio, la cual ha sido valorada de manera positiva por los usuarios. Se está trabajando en la etapa de validación final de sus resultados.

\section{CONCLUSIÓN}

El crecimiento de la COVID 19 en Ecuador ha demandado de manera urgente de la legalización de resoluciones, decretos y normativas de estricta aplicación en a todas las organizaciones, empresas e instituciones de carácter público y privado, para poder hacer frente a esta emergencia sanitaria y de consecuencias letales, una de estas fue la declarada en la mesa técnica del COE Cantonal Guayaquil.

Se hizo imprescindible elaborar la metodología, fases, instrumentos y formas de aplicación y evaluación de la efectividad de las medidas implementadas en los diferentes niveles, a través de la propuesta de una lista de comprobación de los protocolos basada en las normativas establecidos por los organismos de control frente a los 
agentes biológicos, cuyo objetivo es precisamente permitir verificar el grado de cumplimiento de los criterios técnicoslegales establecidos en los protocolos de bioseguridad, acorde a las exigencias emitidas por los entes de control.

El alcance de la Lista de comprobación de protocolos de bioseguridad propuesta, ha sido desarrollado para el uso de instituciones y empresas del sector público y privado, aplicable a nivel nacional, donde se deberá contemplar los requerimientos legales de cada GAD al momento de realizar la evaluación. La misma está en fase de validación final.

\section{BIBLIOGRAFÍA}

1. Aiken, L. R. (2003). TESTS PSICOLÓGICOS Y EVALUACIÓN. En L. R. Aiken, TESTS PSICOLÓGICOS Y EVALUACIÓN (págs. 364-389). México: PEARSON EDUCACIÓN.

2. APSSTEC, SEK, ASPA, CGPSST. (2020). Protocolo Evaluación de Riesgos Laborales Frente al Nuevo Coronavirus (SARS-CoV-2). Método de Evaluación Semicuantitativa. Quito.

3. Cardona, C., \& Restrepo, A. (s.f.). http://puntosdeencuentro.weebly.com/. Recuperado el 10 de jun de 2020, de http://puntosdeencuentro.weebly.com/uploads/2/2/3/6/22361874/listas_de_chequeo.pdf

4. IESS, Freire, A., Guzmán, F., Camino, F., Martínez, D., Vaca, S., . . Chávez, J. (2020). Guía de Prevención y Actuación Integral Frente al SARS-CoV-2 (COVID-19) en el Ámbito Laboral. Quito.

5. IESS, APSSTEC, Arellano, A., Alvarado, J., Defranc, P., Jacome, G., Oviedo, R., Sánchez, Sh., (2020) guía para la comprobación de protocolos de seguridad frente a exposición de agentes biológicos. Guayaquil

6. IESS, Freire, A., Martínez, D., Moreno, F., Orozco, I., Cárdenas, I., . . Chávez, J. (2020). Guía Orientativa de Retorno al Trabajo Frente a Covid-19. Quito.

7. Ministerio de Salud del Gobierno de Chile. (2009). http://www.bibliotecaminsal.cl/. Recuperado el 10 de jun de 2020, de http://www.bibliotecaminsal.cl/wp/wpcontent/uploads/2016/03/24.pdf

8. Oliva, P. (2009). www.minsal.gob.cl. Recuperado el 10 de jun de 2020, de http://www.minsal.gob.cl/portal/url/item/7cf9e499a55c4cc7e04001011f016c69.pdf

9. Padilla, J. C. (2007). Escalas de medición. En Paradigmas (Vol. 2). Bogotá D.C, Colombia: Corporación Universitaria Unitec

10. Kampf G, Scheithauer S, Lemmen S, Saliou P, Suchomel M. COVID-19-associated shortage of alcohol-based hand rubs, face masks, medical gloves and gowns - proposal for a risk-adapted approach to ensure patient and healthcare worker safety. J Hosp Infect. 2020; 105: 424-7.

11. Black JRM, Bailey C, Przewrocka J, Dijkstra KK, Swanton C. COVID-19: the case for health-care worker screening to prevent hospital transmission. Lancet. 2020; 395: 1418-20.

12. Matrajt $L$, Leung $T$. Evaluating the effectiveness of social distancing interventions to delay or flatten the epidemic curve of coronavirus disease. Emerg Infect Dis. 2020 [En prensa]. doi: 10.3201/eid2608.201093

13. Han G, Zhou YH. Possibly critical role of wearing masks in general population in controlling COVID-19. J Med Virol. 2020 [En prensa]. doi: 10.1002/jmv.25886

14. Fiorino G, Colombo M, Natale C, Azzolini E, Lagioia M, Danese S. Clinician education and adoption of preventive measures for COVID-19: A survey of a convenience sample of general practitioners in Lombardy, Italy. Ann Intern Med. 2020 [En prensa]. doi: 10.7326/M20-1447.

15. Chen C, Zhao B. Makeshift hospitals for COVID-19 patients: where health-care workers and patients need sufficient ventilation for more protection. J Hosp Infect. 2020; 105: 98-9.

16. Chen TY, Lai HW, Hou IL, Lin CH, Chen MK, Chou CC, et al. Buffer areas in emergency department to handle potential COVID-19 community infection in Taiwan. Travel Med Infect Dis. 2020 [En prensa]. doi: 10.1016/j.tmaid.2020.101635.

17. Cortes Díaz José M. Técnicas de prevención de riesgos Laborales. Editorial Tebar, S.L.9a Madrid 2007.

18. Cortez Díaz José M. Seguridad e Higiene del Trabajo. Editorial Alfa Omega. España 2002

19. Denton, Keth. Seguridad Industrial: Administración y Métodos. Editorial Mc Graw Hill. 1985.

20. Grimaldi - Simonds. La Seguridad Industrial: Su administración. Editorial Alfa Omega. México 1996

21. Handley, William. Higiene en el Trabajo. Editorial McGraw Hill.

22. Hernández Zúñiga Alfonso. Seguridad e Higiene Industrial. Editorial Limusa. Noriega. 1a. México 2005.

23. Manual de Seguridad e Higiene de la Facultad de Medicina de México (2005).

24. Manual de seguridad para operaciones en laboratorios de biotecnología. Universidad politécnica de Valencia http://www.sprl.upv.es/msbiotecnologia6.htm

25. Higiene y Sanidad Ambiental, 5: 132-137 (2005). 